\title{
Pseudoaneurysm of the
}

\section{Anterior Interosseous Artery and}

Anterior Interosseous Nerve Palsy.

A Rare Postoperative Complication

of Ulnar Fracture Osteosynthesis:

\section{A Case Report}

Magarakis GEORGEa, Tavlas EMMANOUEL ${ }^{\mathrm{b}}$, Kapsetakis PETROS ,

Stavrakakis M. Ioannis FEBOT ${ }^{c}$, Kalliopi ALPANTAKI ${ }^{a}$

aDepartment of Orthopedics and Trauma, Venizeleion General Hospital of Heraklion, Crete, Greece Leof. Knosou 44, Iraklio 71409

${ }^{\text {b}}$ Department of Vascular Surgery, University Hospital of Heraklion, Crete, Greece

'Department of Orthopedics and Trauma, General Hospital of Agios Nikolaos, Greece

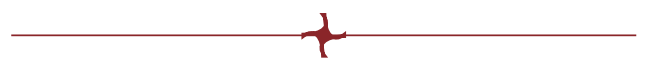

\begin{abstract}
Peripheral arterial pseudoaneurysm is an uncommon complication after open reduction and internal fixation of bone fractures. Here, we describe a rare case of a 72-year-old man who developed a delayed pseudoaneurysm of the anterior interosseous artery after open reduction and internal fixation of an isolated ulnar fracture (nightstick fracture). He presented with a painful forearm mass and anterior interosseous nerve (AIN) neuropathy. Though rare, the appearance of AIN neuropathy is a potential complication of forearm fractures' fixation that should be diagnosed early and managed with a prompt and appropriate treatment.
\end{abstract}

Keywords: pseudoaneurysm, anterior interosseous artery, anterior interosseous nerve palsy, warfarin, forearm fracture, ulna, osteosynthesis.

\footnotetext{
Address for correspondence:

Magarakis George MSc MD

Department of Orthopedics and Trauma, Venizeleion General Hospital of Heraklion, Crete, Greece

Email: magarakisg@gmail.com
} 


\section{INTRODUCTION}

1 pseudoaneurysm is defined as a pulsating, palpable encapsulated hematoma that communicates with the lumen of a ruptured vessel (1). Although a rather common complication of both diagnostic and therapeutic vascular interventions, it has been rarely reported after open reduction and internal fixation (ORIF) with plating of skeletal fractures (2). Here we report a rare case of anterior interosseous artery pseudoaneurysm presented as a painful forearm mass and anterior interosseous nerve (AIN) syndrome in a posttraumatic case of closed isolated ulnar shaft fracture treated by ORIF with plating, three months after the procedure.

\section{CASE PRESENTATION}

72-year-old man suffered an isolated left ulna shaft fracture (nightstick fracture) along with a unilateral humerus shaft fracture and traumatic brain injury after being attacked. At presentation he was neurovascularly intact. His past medical history was positive for receiving warfarin for chronic atrial fibrillation (CAF). The patient underwent ORIF for both upper limb fractures. Pneumatic tourniquet was not used due to the humerus fracture, and according to his surgeon, due to excessive bleeding the surgery was technically demanding. Postoperatively, radial and ulnar pulses were present with normal capillary refill. The patient was discharged and a follow-up was arranged at the outpatient clinic.

One-month postoperatively, the patient gradually developed a tender swelling at the volar site of the forearm, which was initially diagnosed as a local infected hematoma. Despite the fact that the swelling was not located at the site of the surgical wound and the surgery site never showed any infection signs, the swelling was treated with a course of antibiotics. As expected, the swelling showed no signs of improvement and an ultrasound scan of the area was inconclusive. Gradually, the patient developed paresthesia on the index finger. At that time, no actions were taken and another follow-up appointment was arranged after a few weeks.

Almost three months after the operation, the patient came to the emergency department with recurrent swelling and severe pain in the forearm. Upon clinical examination, the same dense, palpable and painful mass located at the proximal volar site of the forearm was identified. Again, no signs of infection were noticed. Sensory deficit of the index finger was present, as described above. Additionally, the patient had gradually developed deteriorating weakness of the flexor pollicis longus ( $\mathrm{FPL}$ ), flexor digitorum profundus (FDP), and pronator quadrates (he was unable to perform the "ok" sign, suggesting
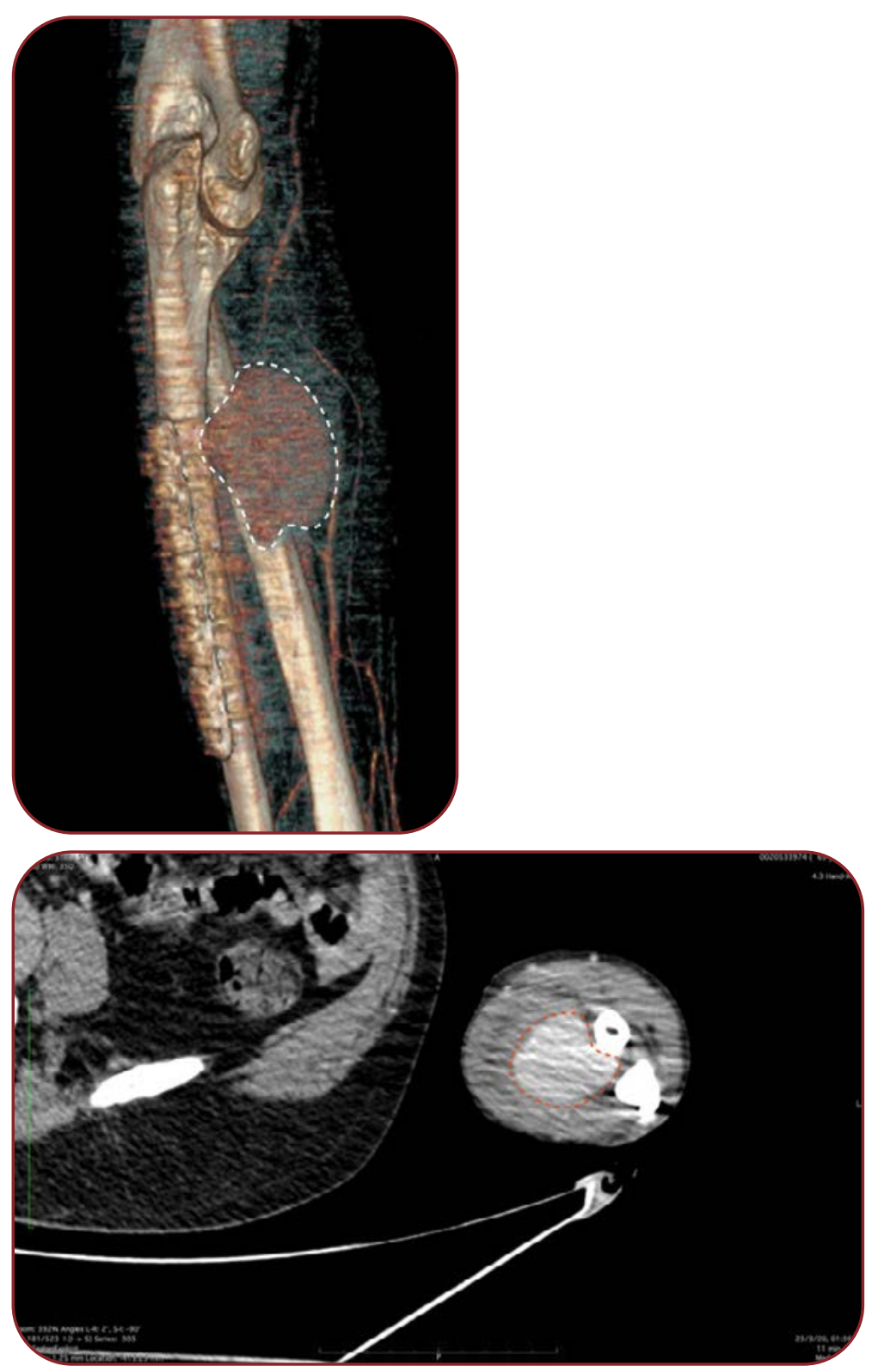

FIGURE 1. 3D CT angiogram reconstruction. Notice that the osteosynthesis is closely related to the pseudoaneurysm. The pseudoaneurysm is depicted with a white dashed line 
AIN palsy). Radial pulse was present but ulnar pulse was not. An 18G needle was introduced into the mass and pulsating blood was aspirated. Arterial blood gas test of the aspirated blood confirmed its arterial origin. The patient was readmitted for further management. A computed tomography angiogram was performed, which displayed a pseudoaneurysm of $5.16 \mathrm{~cm} \times 4.19 \mathrm{~cm}$ approximately of the anterior interosseous artery.

The severe pain and increased risk for pseudoaneurysm rupture, with a subsequent compartment syndrome, led the patient to the operating theater immediately. A pneumatic tourniquet was applied and a proximal Henry approach was performed under general anesthesia. The cyst of the pseudoaneurysm was recognized and opened, the hematoma was drained and the neck of the pseudoaneurysm was identified and ligated. Further examination for active hemorrhage was negative and the wound was sutured. Immediately after surgery, both radial and ulnar pulses were present and low-molecular weight heparin (LMWH) was implemented to warfarin until therapeutic levels of warfarin were achieved, (INR >2). The next day, the patient presented no pain but the AIN palsy remained unchanged. Physiotherapy and active range of motion exercises initiated at the second day postoperatively. Clinical reevaluation was arranged four and ten days after surgery. An ultrasound showed a small, organized hematoma at the operation site. Palpable radial and ulnar pulses were present. The sensory neurological deficit was partially improved, but motor dysfunction was still present.

\section{DISCUSSION}

n this article we present a patient with a late development of AIN syndrome due to a pseudoaneurysm of the anterior interosseous artery, following ORIF of an ulnar nightstick fracture. This case emphasizes the potential vascular and neurological risks of the forearm bone fractures' osteosynthesis, points out the errors in patient's management and underlies the need of high index of suspicion of this rare complication.

latrogenic arterial pseudoaneurysms are quite common complications of the widespread diagnostic and therapeutic intravascular interven- tions (2-4), but have been rarely reported after osteosynthesis of long bone fractures. In fact, most clinical reports describe cases of pseudoaneurysms as consequences of acute penetrating injuries rather than as complications following bone fractures' osteosynthesis $(3,5)$.

In 2017, Dahapute et al published a case of pseudoaneurysm of radial artery after internal fixation of radius shaft fracture two weeks after the operation, treated with surgical excision and end-to-end anastomosis. The authors concluded that the pseudoaneurysm was the result of intraoperative trauma to artery and pointed out that a high index of suspicion must be maintained about the occurrence of this complication secondary to both trauma and surgery (6).

The time for the diagnosis seems to vary, depending on the location of the pseudoaneurysm and the clinical presentation. A delay in the diagnosis is not rare though. In 2016, Ghaffari et al reported a pseudoaneurysm of the posterior tibial artery after tibial plate removal in an 18-year-old patient who developed pain, mild paresthesia and swelling of the affected area four weeks after the operation; however, diagnosis and surgical treatment were delayed several months (7). Similarly, our patient was initially misdiagnosed as suffering from postoperative infection and received a course of antibiotics.

A pulsatile mass, pain, swelling, bruit, vascular and neurologic deficit comprise the predominant presenting symptoms and sings of this clinical entity. However, pulselessness should not rule out the diagnosis, since large hematoma formation may conceal the underlying pseudoaneurysm and its pulsations (7). Nerve compression due to pseudoaneurysm causing peripheral neurological deficit is extremely rare. Furthermore, injuries of the AIN represent only $1 \%$ of nerve injuries of the upper limb. Albeit, AIN syndrome has been previously described after a false aneurysm of the interosseous artery in a case of a minimal penetrating injury of the forearm (5). To our knowledge, this is the first case of ulnar fracture plating resulting in this complication.

To date, reliable and minimal invasive modalities can be used in order to identify and treat peripheral pseudoaneurysm such as ultrasound sonography (DUPLEX), ultrasound-guided localized compression and ultrasound-guided thrombin injections (6). Our preference for CT angio- 
gram over ultrasound scan was mainly based on the indecisive results of the previously performed ultrasound. Ultrasound-guided endovascular interventions are preferred in cases where the collateral circulation is adequate and they are most favored in cases of small, superficial aneurysms. In cases of compartment syndrome or nerve compression, open intervention is recommended (6).

Regarding pathogenesis, we assume that the cause of the pseudoaneurysm formation was probably an occult vascular injury during complicated surgery. We did not identify any screw tip breaching the vessel; therefore, we believe that a Hohmann retractor or a drill possibly was the cause. We suppose that the AIN palsy could have happened due to pressure caused by the pseudoaneurysm and some recovery is expected. No further nerve exploration was performed, thus nerve-conducted studies would be necessary in case of residual neurological dysfunction over the next months. Undoubtedly, oral warfarin was another aggravating factor preventing hemostasis and contributing to the false aneurysm forming process; hence the management of adult trauma in patients receiving antithrombotics can be particularly challenging (8).

In conclusion, learning points of this case are: (1) although extremely infrequent, postoperative pseudoaneurysm following ulnar nightstick fracture and associated neurological deficit can occur even several weeks after the procedure, (2) it may follow a prolonged occult course with confusing clinical presentation misleading the diagnosis (3) surgical treatment is efficient and (4) high index of suspicion must be maintained by the surgeons regarding this rare complication.

Conflicts of interest: none declared. Financial support: none declared.

\section{R EFerences}

1. Sueyoshi E, et al. Visceral and peripheral arterial pseudoaneurysms. AJR Am J Roentgenol 2005;185:741-749. ISSN 0361-803X. Disponível em: < https://www.ncbi.nlm.nih.gov/ pubmed/16120928 >

2. Luther A, Kumar A, Negi KN. Peripheral Arterial Pseudoaneurysms-a 10-Year Clinical Study. Indian J Surg 2015;77(Suppl 2):603-607. ISSN 0972-2068. Disponível em: < https://www.ncbi.nlm.nih.gov/ pubmed/26730072 >.

3. Pini R, et al. False aneurysm of the interosseous artery and anterior interosseous syndrome--an unusual complication of penetrating injury of the forearm: a case report.

J Orthop Surg Res 2009;4:44. ISSN 1749-799X. Disponível em:
< https://www.ncbi.nlm.nih.gov/ pubmed/20034382 >.

4. Stavrakakis IM, et al. Sciatic Nerve Palsy due to a Superior Gluteal Artery Pseudoaneurysm PostBone Marrow Biopsy: A Case Report and Review of the Literature. J Orthop Case Rep 2018;8:74-77. ISSN 2250-0685. Disponível em: $<$ https://www.ncbi.nlm.nih.gov/ pubmed/30687669 >.

5. Komura S, et al. Forearm Compartment Syndrome Concomitant with Pseudoaneurysm of the Anterior Interosseous Artery after Minor Penetrating Injury. J Hand Surg Asian Pac Vol 2018;23:395-398. ISSN 2424-8363. Disponível em: $<$ https://www.ncbi.nlm.nih.gov/ pubmed/30282546 $>$.

6. Dahapute AA, et al. Radial Artery
Pseudoaneurysm in a Post-operative Case of Midshaft Radius Fracture. J Orthop Case Rep 2017;7:3-5. ISSN 2250-0685. Disponível em: < https://www.ncbi.nlm.nih.gov/ pubmed/29600199 >.

7. Ghaffari S, et al. Pseudoaneurysm of the Posterior Tibial Artery following Removal of Tibial Plate- A Rare Case. J Orthop Case Rep 2016;6:46-49. ISSN 2250-0685. Disponível em: $<$ https://www.ncbi.nlm.nih.gov/ pubmed/28116268 >.

8. Wong $\mathrm{H}$, et al. Antithrombotics in trauma: management strategies in the older patients.

J Blood Med 2017;8:165-174.

ISSN 1179-2736. Disponível em: $<$ https://www.ncbi.nlm.nih.gov/ pubmed/29042825 > 